\title{
Mécanismes de la peroxydation lipidique et des anti-oxydations
}

\section{Josiane CILLARD \\ Pierre CILLARD}

Laboratoire de biologie cellulaire et végétale, UPRESS EA 3891 "Groupe de Recherche en Thérapeutique Anticancéreuse, GRETAC», Faculté de pharmacie, Rennes

\begin{abstract}
Lipid peroxidation is a general phenomenon which takes place in foods as well as in cellular membranes and lipoproteins. It leads to oxidative dammages with consequences to food preservation and to the development of various diseases. The targets of lipid oxidation are the polyunsaturated fatty acids. Owing to the fact that oxygen can exist under 2 states, a ground state $\left({ }^{3} \mathrm{O}_{2}\right)$ and an excited state $\left({ }^{1} \mathrm{O}_{2}\right)$, lipid peroxidation can proceed by 2 different reactions. One which involves $\left({ }^{3} \mathrm{O}_{2}\right)$, is a free-radical chain reaction with 3 steps (initiation by free radicals, propagation and terminaison), the other which involves $\left({ }^{1} \mathrm{O}_{2}\right)$ is a fast reaction with direct addition of oxygen to the double bonds of fatty acids. Both reactions lead to the formation of hydroperoxides as primary products. Hydroperoxides decomposed rapidly to give many secondary products such as lipid free radicals which contribute to increase the oxidation of other molecules such as proteins, nucleic acids and other lipids. Ketones, alkanes (pentane, ethane) and aldehydes (MDA, 4HNE) are also formed by hydroperoxide decomposition. Aldehydes are reactive compounds which bind to the amino group of proteins, nucleic acids and lipids causing dammages to these molecules. The consequences for membranes are alterations of their functions which could lead to cell death. In food hydroperoxide decomposition give rise to qualitative alterations. Lipid peroxidation is a major problem for food industry as well as for human health since it is associated to many diseases. Antioxidants can prevent or delay lipid peroxidation by different mechanisms.
\end{abstract}

Key words: unsaturated fatty acids, triplet oxygen, singlet oxygen, free radicals, hydroperoxides, antioxidants

cardiolipines. La lipoperoxydation des membranes va altérer leur fonctionnalité (modification de leur perméabilité, de leur fluidité, perte d'activité d'enzymes, de récepteurs...). L'oxydation des cardiolipines de la mitochondrie est un facteur déterminant dans le déclenchement de l'apoptose des cellules [1]. Les lipoprotéines telles que les LDL, riches en cholestérol et en phopholipides sont également des cibles privilégiées de la peroxydation lipidique. Les LDL oxydées sont fortement incriminées dans l'athérogenèse. De nombreuses autres pathologies sont associées à la peroxydation des lipides. C'est le cas des maladies neurodégénératives (Alzheimer, Parkinson), du diabète, des cancers, des maladies inflammatoires, du vieillissement...

\section{L'autoxydation des acides gras polyinsaturés (AGPI)}

L'autoxydation est une réaction directe de l'oxygène moléculaire avec des composés organiques. Dans le cas des acides gras insaturés, l'oxygène se fixe sur les doubles liaisons pour donner des hydroperoxydes. L'oxygène moléculaire à la particularité d'être un diradical avec 2 électrons non appariés à l'état fondamental dit état triplet $\left({ }^{3} \mathrm{O}_{2}\right)$. L'oxydation directe des acides gras insaturés par ${ }^{3} \mathrm{O}_{2}$ est impossible pour des raisons de spins opposés entre les lipides et ${ }^{3} \mathrm{O}_{2}$. Pour surmonter cette barrière, une étape d'initiation par des radicaux libres est nécessaire pour permettre la fixation d'oxygène sur l'acide gras. L'oxygène peut exister sous une forme beaucoup plus réactive correspondant à un état excité appelée oxygène singulet $\left({ }^{1} \mathrm{O}_{2}\right)$. À la différence de l'oxygène à l'état triplet, l'oxygène singulet se fixe directement sur les doubles liaisons de l'acide gras pour donner des hydroperoxydes.

\section{Autoxydation des ACPI par l'oxygène triplet $\left({ }^{3} \mathrm{O}_{2}\right)$}

La caractéristique de l'autoxydation des AGPI par l'oxygène à l'état fondamental $\left({ }^{3} \mathrm{O}_{2}\right)$ est d'être une réaction en chaîne radicalaire qui se déroule en trois étapes: initiation, propagation, terminaison.

\section{Initiation}

C'est l'abstraction d'un atome d'hydrogène $(\mathrm{H})$ de l'acide gras situé sur un carbone placé entre deux doubles liaisons (liaison hydrogènecarbone plus faible), ce qui conduit à la formation d'un radical alkyle (réaction 1) (figure 1). La réaction d'initiation est induite par les radicaux libres à condition que le potentiel redox 


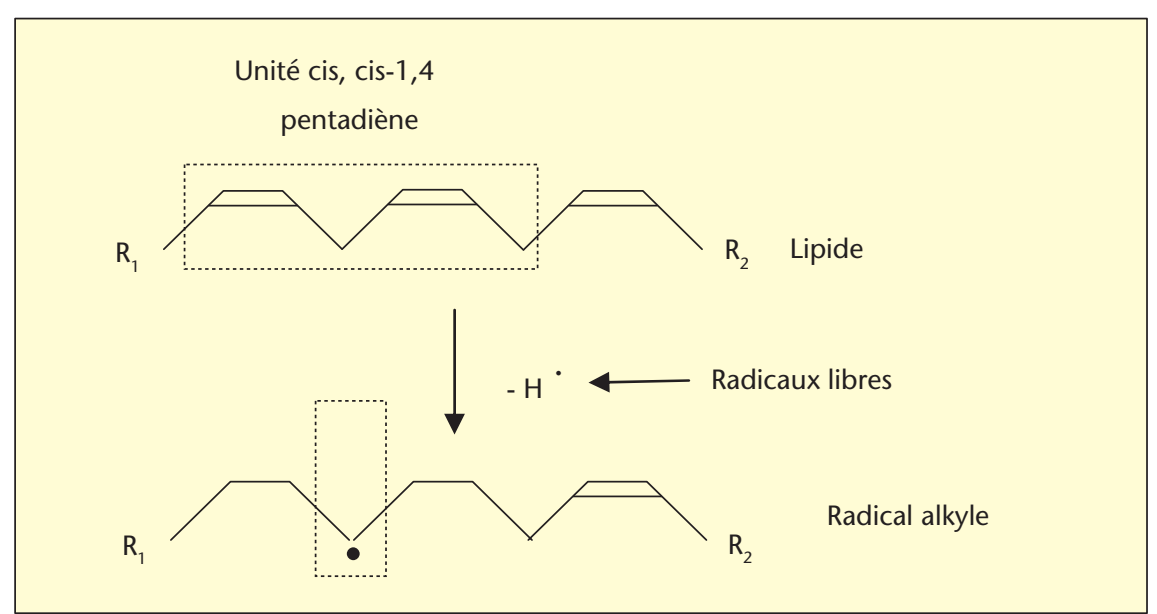

Figure 1. Initiation.

du radical soit supérieur à celui de l'acide gras (un radical pouvant être à la fois oxydant ou réducteur selon les substrats considérés). Les radicaux qui vont initier la peroxydation des AGPI sont principalement les radicaux hydroxyle $(\cdot \mathrm{OH})$ et hydroperoxyle $(\mathrm{HOO} \cdot)$ ainsi que les radicaux lipidiques tels que les radicaux alcoxyle (LO) et peroxyle LOO). Le radical hydroxyle est de loin celui qui a la vitesse de réaction la plus élevée avec les lipides. Sa constante de réaction est de $5.10^{9} \mathrm{M}^{-1} \mathrm{~S}^{-1}$ avec les lécithines [2].

Les sources de radicaux libres sont nombreuses. Il existe deux grandes voies de formation des radicaux libres : le transfert d'électrons catalysé par les métaux de transition (fer, cuivre) et par les enzymes. Ces réactions ont lieu in vitro et in vivo. Les aliments comme les systèmes biologiques (cellules, plasma) contiennent du fer sous forme hémique (hémoglobine, myoglobine) et non hémique. Ce dernier est souvent lié à des petites molécules (ADP, ATP, citrate) et est appelé fer de bas poids moléculaire. II joue le rôle de catalyseur dans le transfert d'électrons sur l'oxygène selon les réactions $2,3,4,5$.

$\mathrm{O}_{2}+\mathrm{Fe}^{2+} \rightarrow \mathrm{O}_{2}^{-\bullet}+\mathrm{e}^{-} \rightarrow \mathrm{H}_{2} \mathrm{O}_{2}$

$\mathrm{H}_{2} \mathrm{O}_{2}+\mathrm{Fe}^{2+} \rightarrow{ }^{\circ} \mathrm{OH}+{ }^{-} \mathrm{OH}+\mathrm{Fe}^{3+}$

réaction de Fenton

$\mathrm{LOOH}+\mathrm{Fe}^{2+} \rightarrow \mathrm{LO}^{\circ}+{ }^{-} \mathrm{OH}+\mathrm{Fe}^{3+}$

$\mathrm{LOOH}+\mathrm{Fe}^{3+} \rightarrow \mathrm{LOO}^{\circ}+\mathrm{H}^{+}+\mathrm{Fe}^{2+}$

Les enzymes qui participent au métabolisme cellulaire tel que la respiration, la synthèse des prostaglandines et des leucotriènes, le catabolisme des bases puriques ou encore le métabolisme des xénobiotiques, l'activation des phagocytes au cours de la réaction inflammatoire, vont permettre la réduction monoélectronique de l'oxygène pour générer des radicaux libres selon la réaction 6 sensibilisateurs (chlorophylle, porphyrine fla vine), au cours d'une réaction dite de type I,

Figure 2. Propagation. des radicaux $\mathrm{O}_{2}^{-\cdot}$ qui donneront à leur tour des radicaux $\cdot \mathrm{OH}$ via les réactions 6 et 3 .

La chaleur provoque la dissociation thermique des hydroperoxydes lipidiques pour donner 'OH et LO' (réaction 7).

$$
\underset{\text { chaleur }}{\rightarrow} \mathrm{LO}^{\circ}+{ }^{\circ} \mathrm{OH}
$$

Il est à noter que les lipides contiennent toujours des traces d'hydroperoxydes qui seront décomposés par les facteurs mentionnés ci-dessus pour donner les radicaux libres initiateurs de la peroxydation lipidique.

\section{Propagation}

Le radical alkyle formé dans l'étape d'initiation réagit très rapidement avec ${ }^{3} \mathrm{O}_{2}$ $\left(\mathrm{K}=10^{9} \mathrm{M}^{-1} \mathrm{~S}^{-1}\right)$ pour donner un radical peroxyle (figure 2). Cette étape est très rapide comparée à la réaction suivante où il y a transfert d'un atome d'hydrogène d'une molécule $d^{\prime}$ acide gras voisine pour former un hydroperoxyde. C'est l'étape de propagation de la peroxydation car l'acide gras qui vient de donner son hydrogène est transformé en radical alkyle qui retourne dans le cycle pour réagir avec ${ }^{3} \mathrm{O}_{2}$ et donner un radical peroxyle. II a été calculé que lorsqu'un radical initie la peroxydation, environ 25 molécules d'acides gras sont oxydées dans la phase de propagation [3]. II est à noter que l'oxydabilité des acides gras augmente avec le nombre de doubles liaisons.

De nombreux isomères $d$ 'hydroperoxydes sont formés dans cette étape. Ainsi l'autoxydation de l'acide linolénique, conduit à des isomères

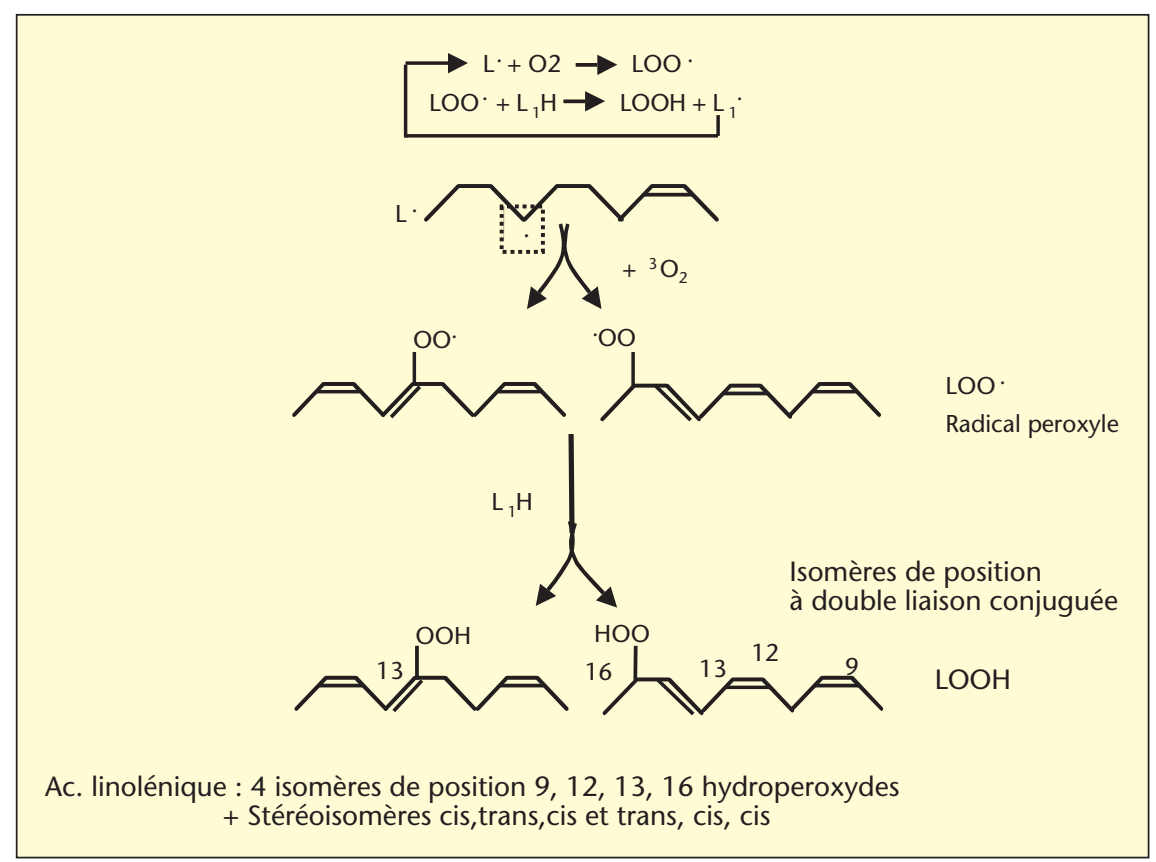


de position $9,12,13,16$, hydroperoxydes. Une stéréo-isomèrie cis, trans, cis et trans, cis, cis s'ajoute à l'isomérie de position. L'acide linoléique donne par autoxydation 2 isomères de position et l'acide arachidonique 6 isomères. Tous ces isomères contiennent des doubles liaisons conjuguées ce qui permet leur détection en UV. En outre des peroxydes cycliques peuvent se former ainsi que d'autres produits comme les isoprostanes qui sont les marqueurs de la peroxydation lipidique in vivo considérés comme les plus fiables actuellement.

\section{Terminaison}

Après avoir atteint une vitesse maximale $d^{\prime} o x y$ dation, celle-ci diminue par combinaison des radicaux lipidiques pour donner des produits non radicalaires. La réaction la plus fréquente est une réaction de dimèrisation qui est liée à la pression partielle d'oxygène du milieu. À pression d'oxygène élevée se sont surtout les radicaux peroxyles qui réagissent entre eux (réaction 8).

$$
\begin{aligned}
\mathrm{LOO}^{\circ}+\mathrm{LOO}^{\circ} \rightarrow & \mathrm{LOOL}+{ }^{1} \mathrm{O}_{2} \\
& \text { produits non radicalaires }
\end{aligned}
$$

II est à noter que certains antioxydants vont provoquer la terminaison de la peroxydation en piégeant les radicaux peroxyles (réaction 9). Ces antioxydants sont appelés «chain breaking "

$$
\mathrm{LOO}^{\circ}+\mathrm{AH} \rightarrow \mathrm{LOOH}+\mathrm{A}^{\circ}
$$

\section{Autoxydation des AGPI par l'oxygène singulet $\left({ }^{1} \mathrm{O}_{2}\right)$}

Les sources d'oxygène singulet sont nombreuses comme l'indique la figure 3. Sa formation est souvent couplée avec la présence d'un photosensibilisateur. Ce dernier qui est à l'état triplet, absorbe des photons et passe à l'état singulet. II transmet son excitation à l'oxygène moléculaire qui devient excité à son tour pour donner l'oxygène singulet qui est environ 1500 fois (forme $\Delta g^{1} \mathrm{O}_{2}$ ) plus réactif que l'oxygène triplet (réaction 10).

$$
{ }^{3} \text { sens }+\mathrm{h} v \rightarrow{ }^{1} \text { sens }+{ }^{3} \mathrm{O}_{2} \rightarrow{ }^{1} \mathrm{O}_{2}
$$

L'oxygène singulet s'additionne directement sur les doubles liaisons des acides gras pour donner des hydroperoxydes (figure 4). Dans le cas de l'acide linoléique, 4 isomères de position sont formés, au lieu de $2 \operatorname{avec}^{3} \mathrm{O}_{2}$. Les isomères 9 et de 13 représentent chacun $30 \%$ et les isomères 10 et 12 , spécifiques de ${ }^{1} \mathrm{O}_{2}$, représentent chacun $20 \%$ [4]. En fait l'autoxydation des acides gras par ${ }^{1} \mathrm{O}_{2}$ est vraisemblablement mineure par rapport à l'autoxydation en chaîne radicalaire $\mathrm{par}{ }^{3} \mathrm{O}_{2}$. Elle fournirait principalement les hydroperoxydes qui par décomposition en radicaux libres serviraient $d$ 'initiateurs de la réaction d'autoxydation $\operatorname{par}^{3} \mathrm{O}_{2}$. II est

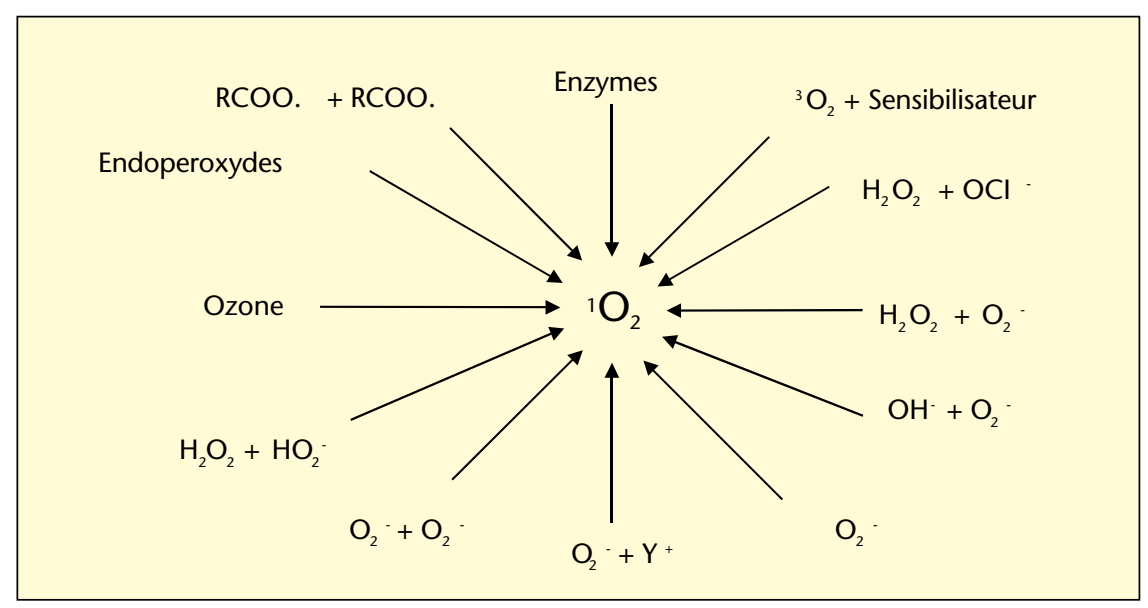

Figure 3. Formation de l'oxygène singulet (d'après Norman Krinsky). noter que la durée de vie de ${ }^{1} \mathrm{O}_{2}$ est plus longue en milieu hydrophobe qu'en milieu aqueux.

L'ensemble des mécanismes d'autoxydation des acides gras est porté dans la figure 5 .

\section{Conséquences des peroxydations lipidiques}

Elles ont très nombreuses. Les hydroperoxydes instables en se décomposant vont donner de nouveaux radicaux libres provoquant des oxydations des biomolécules, mais aussi des aldéhydes réactifs (MDA, 4HNE) qui feront des adduits sur les groupements $\mathrm{NH}_{2}$ des biomolécules (acides nucléiques, protéines, lipides). L'oxydation des protéines au niveau de leurs fonctions thiols altère leurs structures (misfolding des protéines des $L D L$ ) et leurs fonctions (perte d'activité des enzymes et des recepteurs). Toutes les structures de la cellule seront ainsi touchées et particulièrement les membranes (membranes plasmique, mitochondriale lysosomale). La figure 6 illustre les altérations cellulaires résultant de la lipoperoxydation des membranes. Les composés riches en AGPI (corps gras alimentaires, cosmétiques) subiront des modifications organoleptiques (rancidité, acidité, modification de la couleur avec apparition de brunissements). Des conséquences nutritionnelles vont résulter de l'oxydation de nutriments (disparition des vitamines $\mathrm{A}, \mathrm{E}, \mathrm{C}$, oxydation d'acides aminés). Enfin des composés toxiques (peroxydes, époxydes, aldéhydes

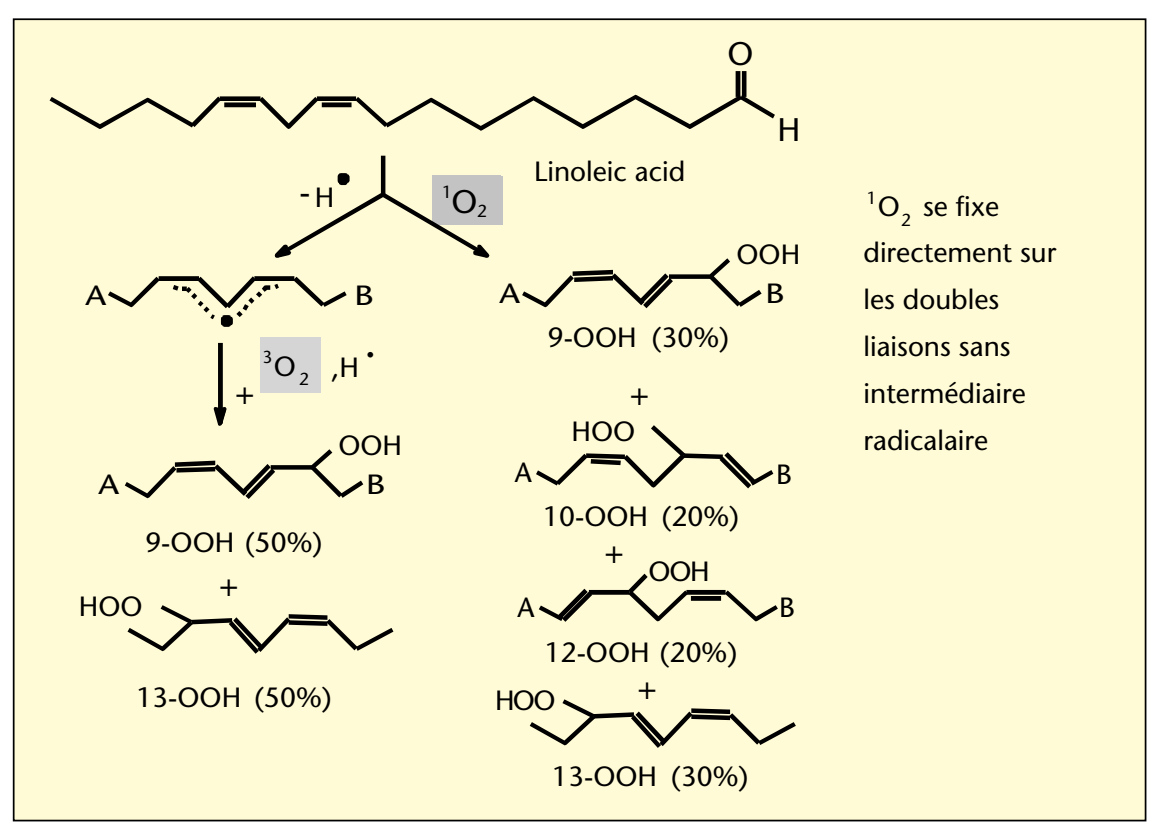

Figure 4. Autoxydation de l'acide linoléique par l'oxygène singulet et l'oxygène triplet. 


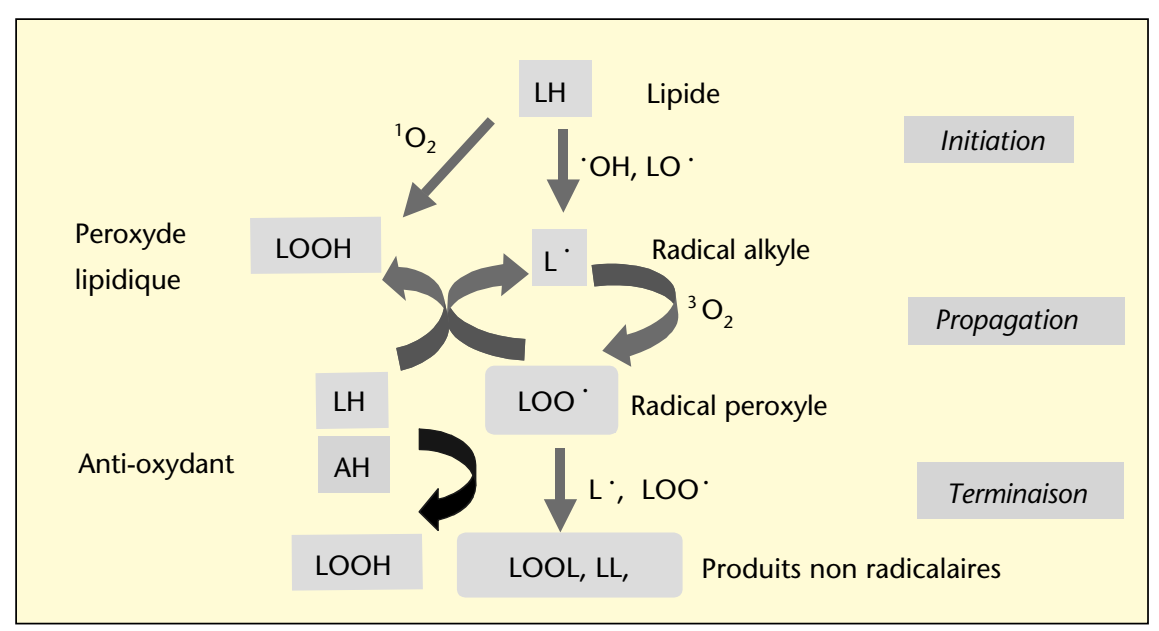

Figure 5. Mécanisme de l'autoxydation des acides gras par l'oxygène triplet et l'oxygène singulet.

mutagènes) vont s'accumuler dans les aliments. La toxicité de ces composés est débattue. Il existe au niveau de la barrière intestinale des systèmes de détoxication. Une glutathion peroxydase spécifique de l'intestin a été identifiée [5]. Elle réduit les hydroperoxydes en dérivés hydroxylés correspondants non toxiques. Les antioxydants vont par conséquent jouer un rôle important dans la prévention et la propagation des dommages oxydatifs.

\section{Mécanismes des anti-lipoperoxydations}

Les antioxydants sont des composés très divers qui regroupent des protéines à activité enzy- matique (superoxyde dismutase, glutathion peroxydase, catalase) et non enzymatique (sequestrant des métaux) et des petites molécules liposolubles (vitamine $E, \beta$-carotène) ou hydrosolubles (vitamine $C$, acide urique). Une définition large du terme antioxydant donnée par B. Halliwell [6] est « toute substance qui, présente à faible concentration comparée à celle du substrat oxydable, retarde ou prévient de manière significative l'oxydation de ce substrat ».

Selon Buettner les antioxydants susceptibles de protéger les lipides de l'oxydation peuvent être répartis en deux types : les antioxydants préventifs qui empêchent la formation d'espèces réac-

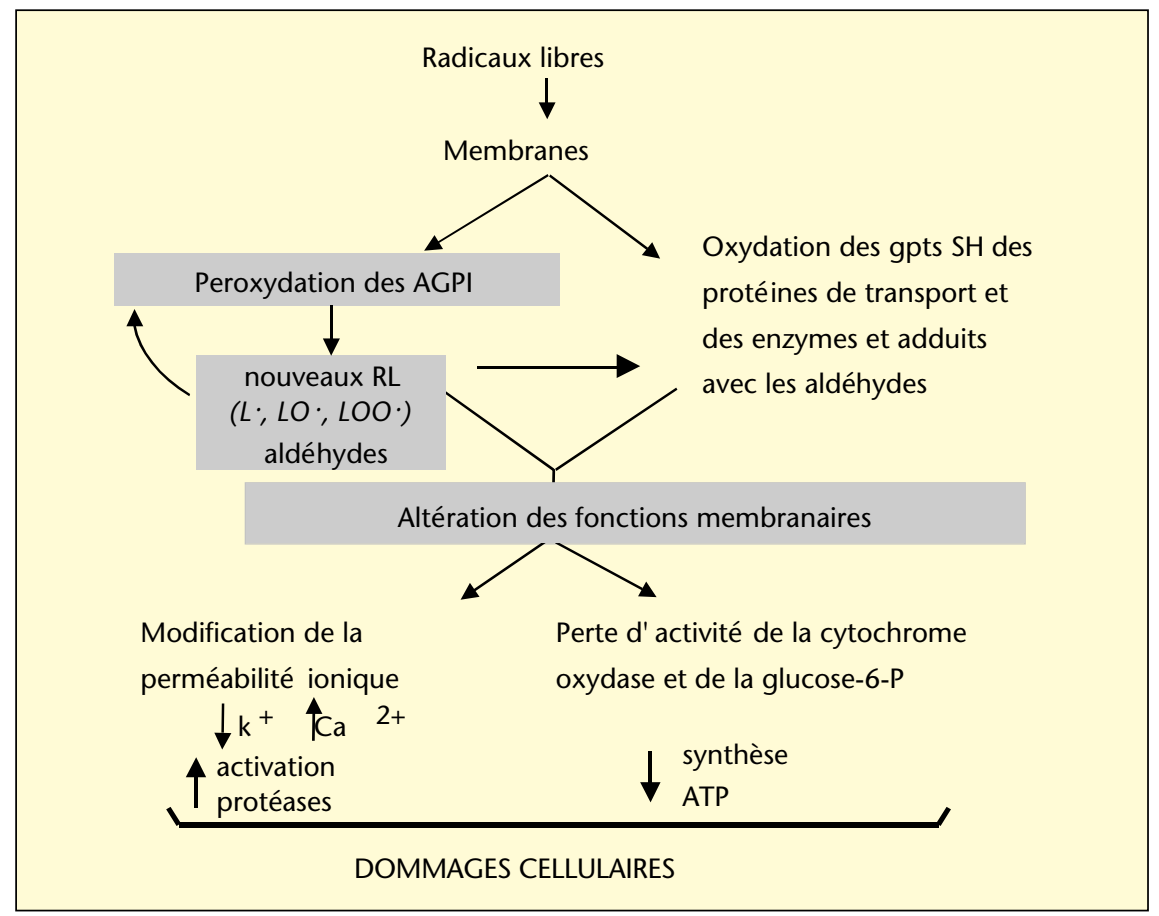

Figure 6. Conséquences cellulaires de la peroxydation lipidique. tives de l'oxygène ou interceptent les espèces responsables de l'initiation de la lipoperoxydation et les antioxydants " chain breaking " qui interceptent les radicaux propagateurs de la peroxydation lipidique et retardent la peroxydation (période d'induction).

\section{Mécanismes d'action des antioxydants préventifs}

- Les chélateurs des métaux de transition comme le fer et le cuivre impliqués dans la génération de radicaux libres (voir les réactions 2 à 5) peuvent prévenir les oxydations. Ces chélateurs forment des complexes ou des composés de coordination avec les métaux. Ils inhibent ainsi le cycle redox du métal ou forment des complexes métalliques insolubles. Ce sont des protéines telles que la transferrine, la ferritine, la lactalbumine qui sequestrent le fer, la céruloplasmine et l'albumine qui sequestrent le cuivre [7]. De nombreuses petites molécules peuvent également chélater ou complexer le fer. C'est le cas des acides phosphorique, citrique, succinique, ascorbique, nitrilotriacétique, phytique (plantes), la desferrioxamine, I'EDTA, le DETAPAC. Certains de ces complexes peuvent être prooxydants $[8,9]$. Les flavonoïdes sont de bons chélateurs du fer ce qui est un des mécanismes de leur activité antioxydante [10, 11].

- Les désactivateurs (quencher) de I'oxygène singulet. Selon Buettner [12], ils peuvent agir par désactivation chimique en se fixant sur une molécule telle qu'un acide gras pour donner un hydroperoxyde (réaction 11) ou encore par désactivation physique éliminant l'énergie d'excitation sans changement chimique (réaction 12).

${ }^{1} \mathrm{O}_{2}+\mathrm{LH} \rightarrow \mathrm{LOOH}$

${ }^{1} \mathrm{O}_{2}+\beta$-carotène $\rightarrow \mathrm{O}_{2}+\beta$-carotène ${ }^{*}$

Les caroténoïdes sont particulièrement efficaces. Le lycopène est le plus réactif. Sa vitesse de réaction avec ${ }^{1} \mathrm{O}_{2}$ est particulèrement élevée $\left(31 \times 10^{9} \mathrm{M}^{-1} \mathrm{~S}^{-1}\right)$ [13]. Le $\beta$ carotène réagit avec ${ }^{1} \mathrm{O}_{2}$ avec une vitesse de réaction 6 fois plus faible $\left(5 \times 10^{9} \mathrm{M}^{-1} \mathrm{~S}^{-1}\right)$. Les tocophérols $\left(2,7 \times 10^{7} \mathrm{M}^{-1} \mathrm{~S}^{-1}\right)$, les thiols, l'acide ascorbique $\left(1 \times 10^{8} \mathrm{M}^{-1} \mathrm{~S}^{-1}\right)$ sont moins efficaces vis à vis de la désactivation de ${ }^{1} \mathrm{O}_{2}$. Néanmoins ces composés ont des concentrations élevées dans les systèmes biologiques et contribuent à égalité avec les caroténoïdes à la désactivation de ${ }^{1} \mathrm{O}_{2}$. Un acide aminé comme I'histidine est aussi un bon « quencher » de ${ }^{1} \mathrm{O}_{2}$.

- L'élimination des hydroperoxydes générateurs de radicaux libres est une autre voie de prévention des oxydations. Les hydroperoxydes peuvent être réduits par des enzymes. La glutathion peroxydase (GPx) éliminent les hydroperoxydes organiques et le peroxyde 


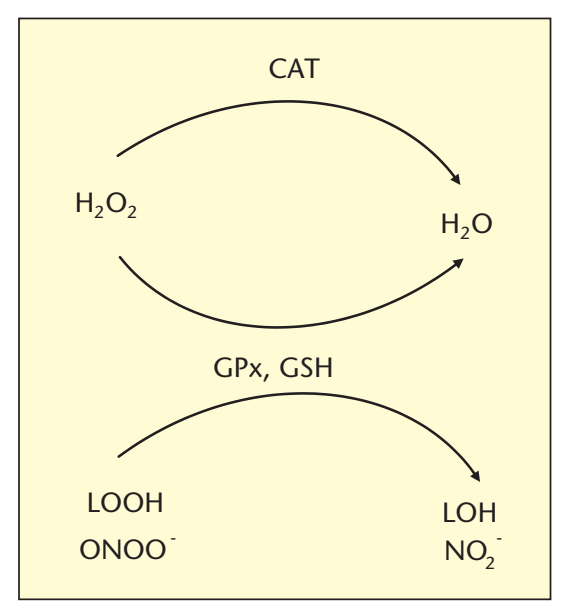

Figure 7. Enzymes impliquées dans l'élimination des hydroperoxydes.

d'hydrogène à faible concentration. Elle élimine également les peroxynitrites qui sont des oxydants très puissants. Elle est présente dans les cellules (cytoplasme, mitichondrie, membranes). Une GPx intestinale a été identifiée récemment [5]. Elle éliminerait les hydroperoxydes provenant des aliments oxydés. Une autre enzyme, la catalase (CAT) a pour unique substrat le peroxyde d'hydrogène qu'elle réduit en eau. L'inconvénient de cette enzyme est qu'elle est localisée uniquement dans les peroxysomes et ne peut donc pas agir dans les autres compartiments cellulaires (cytoplasme, mitochondrie, lysosome, noyau) où le peroxyde d'hydrogène est également présent. Ce dernier diffuse très facilement à travers les membranes et peut donc agir à distance de son lieu de production. Un schéma de l'action des enzymes antioxydantes est porté figure 7.

Outre les enzymes, une molécule comme I'acide pyruvique élimine le peroxyde d'hydrogène pour donner de l'acétate et du dioxyde de carbone. II est à noter que la présence fréquente de pyruvate dans les milieux de cultures cellulaires doit être prise en compte par les expérimentateurs qui utilisent le peroxyde $\mathrm{d}$ 'hydrogène pour induire un stress oxydant.

- Les piègeurs d'oxygène. Ce sont des molécules telles que les sulfites ou l'acide ascorbique.

\section{Mécanismes d'action des antioxydants "chain breaking »}

Cette catégorie d'antioxydants va réagir le plus souvent avec les radicaux peroxyles ou alcoxyles, interrompant ainsi la réaction de propagation de la peroxydation (figure 5). II est à noter que ces antioxydants n'inhiberont pas par conséquent l'autoxydation des lipides par l'oxygène singulet. Ces antioxydants peuvent agir selon deux mécanismes:

- les donneurs d'hydrogène. C'est le cas le plus fréquent. Ils doivent avoir un potentiel de réduction inférieur au potentiel des AGPI $(E=0,6 \mathrm{~V})$ et doivent pouvoir donner un hydrogène au radical alcoxyle $(E=1,6 \mathrm{~V})$ et au radical peroxyle $(E=1 \mathrm{~V}$ ) (figures 8,9$)$. Ces antioxydants sont principalement des composés phénoliques mono- ou polyhydroxylés (tocophérols, tocotriènols, BHT, BHA, flavonoïdes...) avec diverses substitutions sur les noyaux. Après la réaction d'oxydation (figure 9), l'antioxydant est transformé en un radical qui doit être suffisamment stable pour inhiber la formation d'un autre radical et arrêter ainsi la propagation de la chaîne radicalaire. II doit ensuite évoluer vers un produit d'oxydation stable, ce qui conduit à la consommation de I'antioxydant $[9,14]$. Le tocophérol donnera un radical tocophéroxyle (figure 9) qui évoluera vers un composé d'oxydation non radicalaire tel que la tocophérylquinone ou un composé de dimérisation ou de polymérisation supérieure. Ces antioxydants introduisent une phase de latence ou "lag phase » pendant laquelle la peroxydation lipidique est très faible et qui persiste tant que l'antioxydant n'est pas consommé par les radicaux peroxyles. Après la disparition complète de l'antioxydant, la vitesse de la peroxydation augmente très rapidement. II existe un système de recyclage des antioxydants entre eux en fonction de leur potentiel d'oxydo-réduction (figure 8). Ainsi I'acide ascorbique est bien connu pour recycler le tocophérol.

- Les antioxydants "sacrifiés". Le qualifiquatif de « chain breaking antioxidant sacrificial » employé par Buettner concerne des molécules, elles mêmes radicalaires, qui réagissent avec les radicaux peroxyles ou alcoxyles pour donner des produits non radicalaires interrompant ainsi la propagation de la peroxydation. Deux radicaux sont connus pour se combiner avec les radicaux peroxyles: le monoxyde d'azote (NO) et l'anion superoxyde $\left(\mathrm{O}_{2}^{-}\right)$.

Le NO est produit par voie enzymatique dans les cellules grâce à une NO synthase mais il peut se former également à partir des nitrites et des nitrates. NO réagit avec les radicaux peroxyles (réaction 13) et alcoxyles (réaction 14) pour donner des composés d'addition non radicalaires.

$$
\begin{gathered}
\mathrm{LOO}^{\circ}+{ }^{\circ} \mathrm{NO} \rightarrow \text { LOONO } \\
\mathrm{LO}^{\circ}+\mathrm{NO} \rightarrow \text { LONO }
\end{gathered}
$$

\begin{tabular}{|lc|}
\hline \multicolumn{1}{c|}{ Composés } & $E(\mathrm{mV})$ \\
\hline $\mathrm{HO} \cdot, \mathrm{H}^{+} / \mathrm{H} \mathrm{O}$ & 2310 \\
$\mathrm{LO} \cdot \mathrm{H}^{+} / \mathrm{LOH}$ & 1600 \\
$\mathrm{LOO} \cdot \mathrm{H}^{+} / \mathrm{LOOH}$ & 1000 \\
$\mathrm{~L} \cdot \mathrm{H}^{+} / \mathrm{LH}$ & 600 \\
Catéchol $\cdot \mathrm{H}^{+} /$Catéchol & 530 \\
$\alpha$-Tocophéroxyle $\cdot \mathrm{H}^{+} / \alpha$-Tocophérol & 500 \\
Ascorbate $\cdot, \mathrm{H}^{+} /$Ascorbate & 282 \\
\hline
\end{tabular}

Figure 8. Potentiels de réduction standard (d'après GR Buettner).

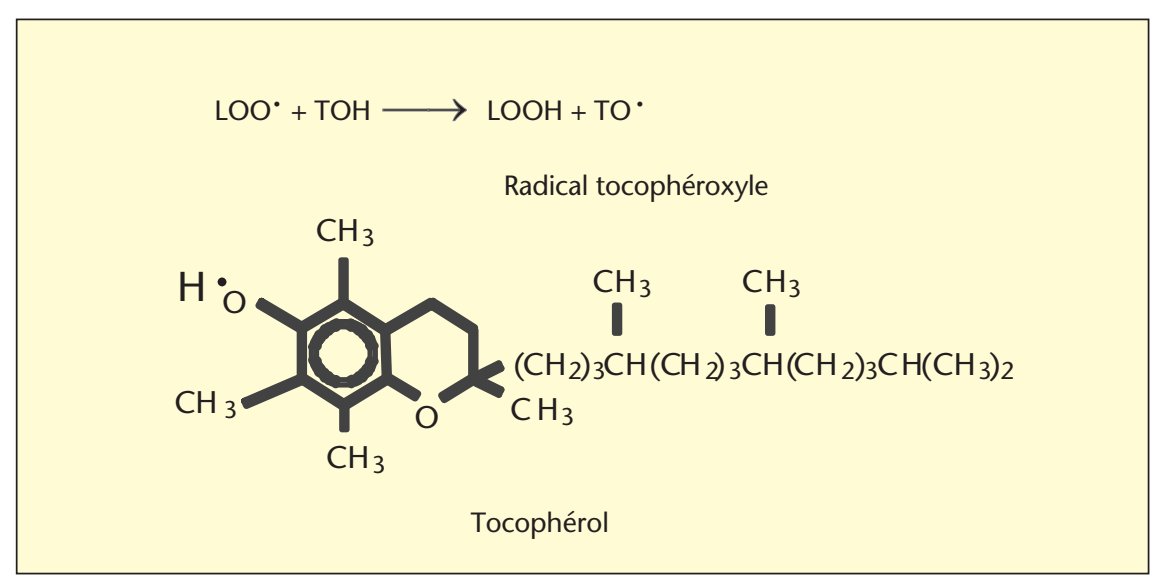

Figure 9. Mécanisme d'action d'un anti-oxydant donneur d'hydrogène comme le tocophérol. 
Récemment il a été montré que l'ingestion alimentaire de petites quantités de nitrates (2 mmoles, ce qui est une quantité normalement présente dans une laitue) produisait une quantité importante de NO dans l'estomac [15, 16] et que ce NO avait un effet protecteur dans les gastrites du à ses effets antioxydants [16]. NO peut aussi agir comme antioxydant préventif en formant des complexes inactifs avec le fer hémique et le fer de bas poids moléculaire (réaction 15). Le fer de bas poids moléculaire correspond au fer lié à des petites molécules comme les citrates, I'ADP, I'ATP dans les cellules.

$$
\mathrm{Fe}^{2+}+{ }^{\circ} \mathrm{NO} \rightarrow \mathrm{Fe}^{2+}-\mathrm{NO}
$$

Une inhibition de la lipoperoxydation par formation de ces complexes a été mise en évidence dans les hépatocytes soumis à un stress oxydant par le fer [17]

L'anion superoxyde peut aussi interrompre la propagation de la peroxydation en réagissant avec les radicaux peroxyles (réaction 16) et alcoxyles (réaction 17).

$$
\begin{gathered}
\mathrm{LOO}^{\circ}+\mathrm{O}_{2}^{-}+\mathrm{H}^{+} \rightarrow \mathrm{LOOH}+\mathrm{O}_{2} \\
\mathrm{LO}^{\circ}+\mathrm{O}_{2}^{-}+\mathrm{H}^{+} \rightarrow \mathrm{LOH}+\mathrm{O}_{2}
\end{gathered}
$$

Ces réactions de terminaison de la lipoperoxydation par l'anion superoxyde expliqueraient le rôle prooxydant des fortes concentrations de superoxyde dismutase [18].

\section{Mécanismes d'action mixtes}

En fait de nombreux antioxydants ont des modes d'action mixtes ce qui complique leur classification. Deux exemples peuvent illustrer ces mécanismes multiples:

- L'acide ascorbique est un désactivateur de l'oxygène singulet, il élimine aussi I'oxygène moléculaire, il est aussi un donneur d'hydrogène aux radicaux lipidiques et aux radicaux tocophéroxyles pour regénérer le tocophérol.

- Les flavonoïdes tels que les anthocyanines, les catéchines, les flavones, les flavonols, les isoflavones et les proanthocyanidines sont chélateurs de métaux, piègeurs d'anions superoxyde et donneurs d'hydrogène.

\section{Les effets prooxydants}

Tout antioxydant peut devenir pro-oxydant. L'effet prooxydant de l'acide ascorbique en présence de fer est bien documenté. De même nous avons mis en évidence un effet prooxydant de l'alpha tocophérol à fortes concentrations $[19,20]$. Les flavonoïdes comme la quercétine et la myricétine peuvent également présenter des propriétés pro-oxydantes [21].

\section{Conclusion}

La peroxydation des lipides est un phénomène qui se produit in vitro et in vivo selon un même mécanisme. Les acides gras insaturés sont la cible des oxydations. L'oxygène moléculaire dans son état fondamental $\left({ }^{3} \mathrm{O}_{2}\right)$ est peu réactif et ne se fixera sur les acides gras qu'après une étape d'initiation induite par les radicaux libres. L'oxygène peut exister sous une forme excitée $\left({ }^{1} \mathrm{O}_{2}\right)$ qui permet une addition rapide et directe de l'oxygène sur l'acide gras.

Les produits primaires résultant de l'oxydation des acides gras sont des peroxydes qui se décomposent rapidement pour donner de très nombreux produits de décomposition qui vont contribuer à propager des dommages vis-à-vis d'autres molécules comme les protéines, les acides nucléiques et d'autres lipides. Du fait des conséquences dommageables de la peroxydation lipidique elle constitue un problème majeur pour l'industrie alimentaire confrontée à la préservation des aliments mais également pour la santé humaine car elle est associée à de nombreuses pathologies. C'est pourquoi les moyens de l'inhiber par les antioxydants sont actuellement un des challenges majeurs de santé publique. Le programme national « Nutrition-Santé » (PNNS) a retenu l'apport d'antioxydants alimentaires (fruits, légumes) parmi ses objectifs prioritaires. L'utilisation de mélange d'antioxydants serait plus efficace (effet de synergie) que celle d'antioxydants pris isolément du fait du recyclage redox des antioxydants entre eux.

\section{RÉFÉRENCES}

1. NAKAGAWA Y. Initiation of apoptotic signal by the peroxidation of cardiolipin of mitochondria. Ann N Y Acad Sci 2004 ; 1011 : 177-84.

2. BUETTNER GR. The pecking order of free radicals and antioxidants: lipid peroxidation, alpha-tocopherol and ascorbate. Arch Biochem Biophys $1993 ; 300: 535-43$.

3. BARCLAY LRC, INGOLD KU. Autoxidation of biological molecules. The autoxidation of a model membrane. A comparison of the autoxidation of egg lecithin phosphatidylcholine in water and chlorobenzene. I Am Chem Soc $1981 ; 103: 6478-85$.

4. FRANKEL EN. Photooxidation of unsaturated fats. In : Frankel EN, ed. Lipid oxidation, the oily press Dundee. 1998 : 43-54.

5. FLORIANS G, WINGLER K, SCHMEHL K, et al. Cellular and subcellular localization of gastro intestinal glutathione peroxidase in normal and malignant human intestinal tissue. Free Radic Res $2001 ; 35: 655-63$.

6. HALLIWELL B. How to characterize a biological antioxidant. Free Radic Res Commun $1990 ; 13$ : $1-32$.

7. MUGGLIR. Free radical tissue damage: the protective rôle of antioxidant nutrients. In : Conrongiu F, Banni S, Dessi MA, Rice-Evans C, eds. Free Radicals and Antioxidants in Nutrition. London : Richelieu press, 1993 : 189-204.
8. BURKITT MJ, GILBERT BC. The autoxidation of iron (II) in aqueous systems : the effects of iron chelation by physiological, non physiological and therapeutic chelators on the generation of reactive oxygen species and the inducement of biomolecular damage. Free Radic Res Commun $1991 ; 14$ : 107-23.

9. HALLIWELL B. Antioxidant characterization, methodology and mechanism. Biochem Pharmacol $1995 ; 49$ : 1341-8.

10. MORELI, LESCOAT G, COGREL P, et al. Antioxidant and iron-chelating activities of the flavonoids catechin, quercetin and diosmetin on iron-loaded rat hepatocytes cultures. Biochem Pharmacol 1993 ; 45 : 13-9.

11. VAN ACKER $S$, BAST A, VAN DER VIJGHW. Stuctural aspects of antioxidant activity of flavonoids. In : Rice-Evans C, Packer L, eds. Flavonoids in health and disease. Marcel Decker New York, 1998 : 221-51.

12. BUETTNER G. Singlet oxygen toxicity is cell line-dependant : a study of lipid peroxidation in nine leukemia cell lines. Photochem Photobiol $1999 ; 70: 858-67$.

13. DI MASCIO P, DEVASAGAYAM TP, KAISER S, SIES H. Carotenoids, tocopherols and thiols as biological singlet molecular oxygen quenchers. Biochem Soc Trans 1990 ; 18 : 1054-6.

14. CILLARD J, CILLARD P. Behavior of alpha, gamma and delta tocopherols with linoleic acid in aqueous media. J Am Oil Chem Soc 1980 ; 57 : 39-42.

15. MCKNIGHT GM, SMITH LM, DRUMOND RS, et al. Chemical synthesis of nitric oxide in the stomach from dietary nitrate in humans. Gut $1997 ; 40: 211-4$.

16. LAROCHE M, ANTON PM, GARCIA-VILLAR R, et al. Protective effect of dietary nitrate on experimental gastritis in rats. Br J Nutr 2003 ; 89 : 777-86.

17. SERGENT O, GRIFFON B, MOREL I, et al. Effect of nitric oxide on iron-mediated oxidative stress in primary rat hepatocyte culture. Hepatology $1997 ; 25: 122-7$.

18. NELSON SK, BOSE SK, MCCORD JM. The toxicity of high-dose superoxide dismutase suggests that superoxide can both initiate and terminate lipid peroxidation in the reperfused heart. Free Radic Biol Med 1994 ; 16 : 195-200.

19. CILLARD J, CILLARD P, CORMIER M, GIRRE L. Alpha tocopherol prooxidant effect in aqueous media : increased autoxidation rate of linoleic acid. J Am Oil Chem Soc 1980 ; 57 : 252-5.

20. CILLARD ], CILLARD P, CORMIER M. Effect of experimental factors on the prooxidant behavior of alpha tocopherol. I Am Oil Chem Soc $1980 ; 57: 255-61$.

21. ARUOMA O. Prooxidant properties : an important consideration for food additives and/or nutrients components? In: Aruoma O, Halliwell B, eds. Free Radicals and Food Additives. Taylor \& Francis London, 1991 : 173-94. 